\title{
Magnetic-Field-Resilient Superconducting Coplanar-Waveguide Resonators for Hybrid Circuit Quantum Electrodynamics Experiments
}

\author{
J.G. Kroll, ${ }^{1,2}$ F. Borsoi, ${ }^{1,2}$ K.L. van der Enden, ${ }^{1,2}$ W. Uilhoorn, ${ }^{1,2}$ D. de Jong, ${ }^{1,2}$ M. Quintero-Pérez, ${ }^{1,3}$ \\ D.J. van Woerkom, ${ }^{1,2}$ A. Bruno,,${ }^{1,2}$ S.R. Plissard,${ }^{4, \dagger}$ D. Car, ${ }^{4}$ E.P.A.M. Bakkers,${ }^{4}$ M.C. Cassidy,${ }^{1,2, \ddagger}$ and \\ L.P. Kouwenhoven ${ }^{1,2,5, *}$ \\ 'QuTech, Delft University of Technology, Delft 2600 GA, Netherlands \\ ${ }^{2}$ Kavli Institute for Nanoscience, Delft University of Technology, Delft 2600 GA, Netherlands \\ ${ }^{3}$ Netherlands Organisation for Applied Scientific Research (TNO), Delft 2600 AD, Netherlands \\ ${ }^{4}$ Department of Applied Physics, Eindhoven University of Technology, Eindhoven 5600 MB, Netherlands \\ ${ }^{5}$ Microsoft Quantum Lab Delft, Delft 2600 GA, Netherlands
}

(Received 12 September 2018; revised manuscript received 26 February 2019; published 24 June 2019)

\begin{abstract}
Superconducting coplanar-waveguide resonators that can operate in strong magnetic fields are important tools for a variety of high-frequency superconducting devices. Magnetic fields degrade resonator performance by creating Abrikosov vortices that cause resistive losses and frequency fluctuations or suppress the superconductivity entirely. To mitigate these effects, we investigate lithographically defined artificial defects in resonators fabricated from $\mathrm{Nb}-\mathrm{Ti}-\mathrm{N}$ superconducting films. We show that by controlling the vortex dynamics, the quality factor of resonators in perpendicular magnetic fields can be greatly enhanced. Coupled with the restriction of the device geometry to enhance the superconductors critical field, we demonstrate stable resonances that retain quality factors $\simeq 10^{5}$ at the single-photon power level in perpendicular magnetic fields up to $B_{\perp} \simeq 20 \mathrm{mT}$ and parallel magnetic fields up to $B_{\|} \simeq 6 \mathrm{~T}$. We demonstrate the effectiveness of this technique for hybrid systems by integrating an In-Sb nanowire into a field-resilient superconducting resonator and use it to perform fast charge readout of a gate-defined double quantum dot at $B_{\|}=1 \mathrm{~T}$.
\end{abstract}

DOI: 10.1103/PhysRevApplied.11.064053

\section{INTRODUCTION}

Superconducting (SC) coplanar-waveguide (CPW) resonators are invaluable tools for parametric amplifiers $[1,2]$, photon detectors that operate from the infrared to x-ray frequencies [3-5] and hybrid systems that couple superconducting circuits to cold atoms [6], solidstate spin ensembles [7-9], nanomechanical resonators $[10,11]$, and semiconducting devices [12-18]. They are also a key component in a variety of quantum-computing (QC) platforms, where they are used for readout, control, and long-range interconnection of superconducting [19-22], semiconducting [23-26], and topological [27,28] qubits.

A common requirement among these schemes is that the $\mathrm{SC}$ resonators be low loss in order to minimize unwanted relaxation via the Purcell effect [29] and maximize two

\footnotetext{
*Leo.Kouwenhoven@Microsoft.com

†Current address: CNRS, LAAS-CNRS, Université de Toulouse, 31400 Toulouse, France

$\ddagger$ Current address: Microsoft Quantum Sydney, Sydney, NSW 2006, Australia
}

qubit gate fidelities [30]. Significant progress has been made in identifying the major loss mechanisms involved. Abundant defects in amorphous materials that couple dissipatively to the electric field of SC CPW resonators and act as effective two-level systems (TLSs) were the first to be identified [31]. Their detrimental effects can be mitigated by minimizing the interface area and improving the quality of interfaces. Subsequently, stray infrared radiation was shown to generate quasiparticles in the SC, significantly increasing losses and necessitating multistage radiative shielding to isolate the resonators from their environment [32]. In addition, when fabricated from type II superconductors, even small magnetic fields $\left(B_{c_{1}} \simeq 1 \mu \mathrm{T}\right)$ result in the creation of Abrikosov vortices: regions of supercurrent that circulate a nonsuperconducting core. When exposed to high-frequency radiation, the Lorentz force causes them to oscillate, generating quasiparticles and increasing losses [33]. Encasing the resonators in multiple layers of magnetic shielding strongly suppresses the number of vortices generated by local magnetic fields, while the use of a type I SC such as Al allows for complete expulsion of magnetic flux via the Meissner effect. Through a combination of these techniques, internal quality factors $\left(Q_{i}\right)$ in excess 
of $10^{6}$ have been demonstrated at single-photon power levels $[34,35]$.

Many semiconducting and topological QC schemes are accompanied by an additional requirement: strong magnetic fields that approach and sometimes exceed $B=1 \mathrm{~T}$. This renders traditional magnetic shielding methods useless and destroys the superconductivity of the $\mathrm{Al} \mathrm{com-}$ monly used to fabricate SC CPW resonators $\left(B_{c} \simeq 10 \mathrm{mT}\right.$ for bulk Al). One possible solution utilizes type II superconductors with high upper critical magnetic fields, such as Mo-Re, TiN, or Nb-Ti-N, allowing the superconductivity to persist in very strong magnetic fields $\left(B_{c_{2}}^{\mathrm{Mo}-\mathrm{Re}}>8 \mathrm{~T}\right.$ [36], $B_{c_{2}}^{\mathrm{Nb}-\mathrm{Ti}-\mathrm{N}}>9 \mathrm{~T}$ [37]) while possessing a low enough density of TLSs to allow high- $Q_{i}$ resonators at zero field $[38,39]$. In order to create high- $Q_{i} \mathrm{SC}$ resonators that can survive in strong magnetic fields, control of the creation and dynamics of Abrikosov vortices is key. Previous studies have utilized the intrinsic disorder in $\mathrm{Nb}-\mathrm{Ti}-\mathrm{N} \mathrm{[40]} \mathrm{and}$ yttrium-barium-copper-oxide (YBCO) [41] films, or lithographically defined artificial defect sites [42] to pin the vortices, preventing dissipation and demonstrating moderate quality factors up to $10^{4}$ in parallel magnetic fields. In other studies, the resonator geometry has been restricted below the superconducting penetration depth to prevent the formation of vortices entirely [43-47]. Impressively, nanowire resonators have achieved $Q_{i}$ of $10^{5}$ at $B_{\|} \simeq 6 \mathrm{~T}$ and $Q_{i}=10^{4}$ at $B_{\perp}=350 \mathrm{mT}$; however, this nonstandard geometry may hinder their implementation into complex systems due to the challenging lithography, the required removal of the ground plane, and the strong sensitivity of the resonator frequency to variations in the film's kinetic inductance. When considering reliability and scalability, devices based on CPW resonators that have been rendered field compatible may be preferable.

In this work, we demonstrate that thin-film Nb-Ti-N SC CPW resonators with lithographically defined artificial defect sites (hereinafter referred to as "holes") can retain a high $Q_{i}$ in strong magnetic fields. We first study how the SC film thickness affects the response and $Q_{i}$ of resonators in parallel magnetic fields in order to optimize field resilience. We then determine how the hole density affects the dynamics of Abrikosov vortices, enabling $Q_{i} \simeq 10^{5}$ to be retained in perpendicular magnetic fields up to $B_{\perp} \simeq$ $20 \mathrm{mT}$. Combining these results, we reduce the number of vortices that are generated by restricting the film thickness and utilize holes to control the vortices that do occur. This allows $Q_{i} \simeq 10^{5}$ at single-photon power levels to be retained up to $B_{\|} \simeq 6 \mathrm{~T}$. Finally, we use these patterned resonators to perform fast charge readout of a hybrid $\mathrm{In}-\mathrm{Sb}$ nanowire double-quantum-dot (DQD) device at $B_{\|}=1 \mathrm{~T}$.

\section{METHODS}

The superconducting CPW resonators are fabricated from $\mathrm{Nb}-\mathrm{Ti}-\mathrm{N}$ films sputtered from a $99.99 \%$ purity $\mathrm{Nb}-\mathrm{Ti}$ target in an $\mathrm{Ar} / \mathrm{N}$ atmosphere onto sapphire (0001 orientation) wafers with a diameter of 2 in and a thickness of $430 \mu \mathrm{m}$. A typical $100-\mathrm{nm}$ film has a $T_{c}$ of $14 \mathrm{~K}$, a resistivity of $123 \mu \Omega \mathrm{cm}$, and a stress of $-400 \mathrm{MPa}$. The resonators are defined using electron-beam lithography (EBL) and a subsequent reactive ion etch (RIE) in an $\mathrm{SF}_{6} / \mathrm{O}_{2}$ atmosphere and, if desired, holes can be patterned and etched in the same step. After fabrication, the samples are mounted in a light-tight copper box and thermally anchored to a dilution refrigerator with a base temperature of $15 \mathrm{mK}$. The complex microwave transmission $S_{21}$ through the sample is measured using standard heterodyne demodulation techniques [Fig. 1(a)], which allow the complex transmission $S_{21}$ to be measured as the probe frequency $f$ is varied. The input line to the sample is heavily attenuated, which suppresses thermal noise and allows the cavity-photon occupancy to reach below a single photon. An external magnetic field is applied to the sample with a three-axis 6-1-1 $\mathrm{T}$ vector magnet. The circulators used in this study are magnetically shielded to prevent stray fields from interfering with

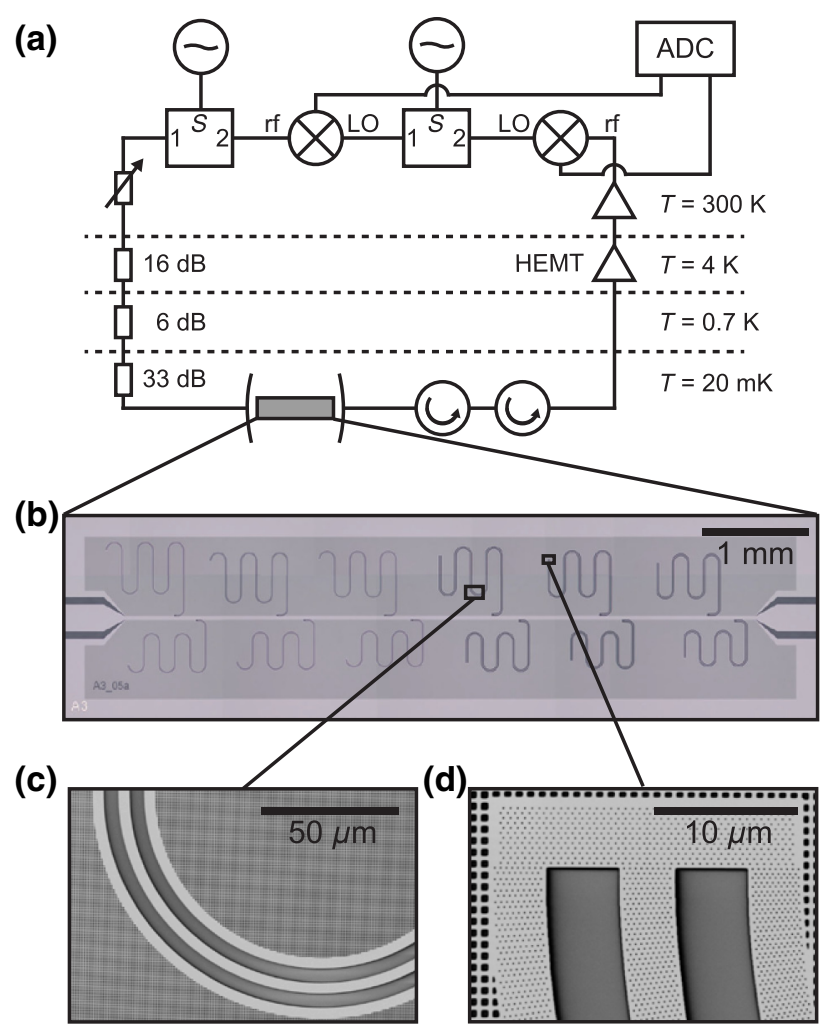

FIG. 1. (a) A diagram of the heterodyne detection circuit, which allows the complex transmission $S_{21}$ to be measured. (b) A cropped optical image showing a typical device with multiple $\lambda / 4$ resonators frequency multiplexed to a common feed line and surrounded by a patterned ground plane. (c),(d) Scanning electron microscope (SEM) micrographs of a SC CPW resonator without (c) and with holes (d) ADC: analog-to-digital converter, HEMT: high-electron-mobility transistor, LO: local oscillator. 
their operation. Two different types of samples are used in this study. The first, used for characterization experiments, contains several $\lambda / 4$ resonators multiplexed to a single feed line [Figs. 1 (b) and 1(d)]. This allows for the coupling quality factor $Q_{c}$ and internal quality factor $Q_{i}$ to be accurately determined by fitting the transmission spectra [48]. In the second geometry, we use a $\lambda / 2$ resonator geometry that is integrated with a nanowire DQD. In this case, we only fit the loaded quality factor $Q_{1}$. All internal quality factors reported in this paper correspond to single-photon power levels. For detailed notes on fitting procedures and device parameters, see the Supplemental Material [49].

\section{FILM-THICKNESS DEPENDENCE}

We first utilize the critical field enhancement observed in thin strips of type II superconductors [43]. Application of a magnetic field along an axis where the thickness $t$ is significantly smaller than the penetration depth $\lambda$ results in complete expulsion of vortices below an enhanced critical field. This enhanced field takes the form $B_{c_{1}}=1.65 \Phi_{0} / t^{2}$, where $\Phi_{0}$ is the magnetic flux quantum and $t$ is the film thickness $[43,44]$. Calculation of $B_{c_{1}}$ for a $22-\mathrm{nm}$ film suggests that the first vortex nucleates at $B_{c_{1}}=7.05 \mathrm{~T}$ for a perfectly aligned field, an improvement of many orders of magnitude compared to a bulk film.

To investigate this effect, a series of SC CPW resonators without holes are fabricated on four different $\mathrm{Nb}-\mathrm{Ti}-\mathrm{N}$ films of thickness $8,22,100$, and $300 \mathrm{~nm}$ [Fig. 2(a)]. $\lambda$ can be estimated in extremely dirty type II superconductors for $T \rightarrow 0 \mathrm{~K}$ as $\lambda=\sqrt{\hbar \rho / \pi \mu_{0} \Delta_{0}}$ [50], where $\hbar$ is the reduced Planck constant, $\rho$ is the film resistivity, $\mu_{0}$ is the permeability of free space, and $\Delta_{0}$ is the superconducting gap at $T=0 \mathrm{~K}$. For our films, $\lambda$ is estimated to vary between 350 and $480 \mathrm{~nm}$ depending on the film thickness and the measured material parameters.

In thin films, the reduction in the Cooper-pair density causes the inertial mass of the charge carriers to become important at microwave frequencies, which results in an additional series inductance or kinetic inductance $L_{\mathrm{k}}$. We estimate the kinetic-inductance fraction $\alpha=L_{k} /\left(L_{g}+L_{k}\right)$ for each film by comparing the measured resonator frequency to one predicted by an analytical model, where $L_{g}$ is the inductance due to the resonator geometry [51]. Thinner films show an increased kinetic inductance fraction, as expected, for a strongly disordered superconductor such as Nb-Ti-N [Fig. 2(b)]. While thinner films should increase the magnetic field compatibility, as $\alpha$ approaches 1 the increase in inductance adds additional challenges for impedance matching and frequency targeting. Moreover, frequency targeting of the resonators becomes increasingly difficult due to the sensitivity of $L_{k}$ with respect to material parameters that become less consistent in thinner films [50]. (a)

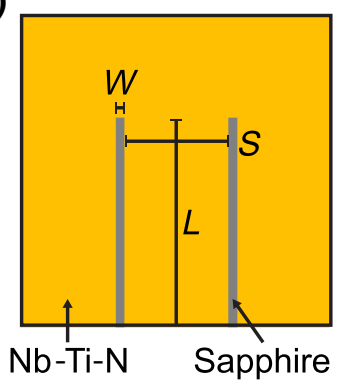

(b)
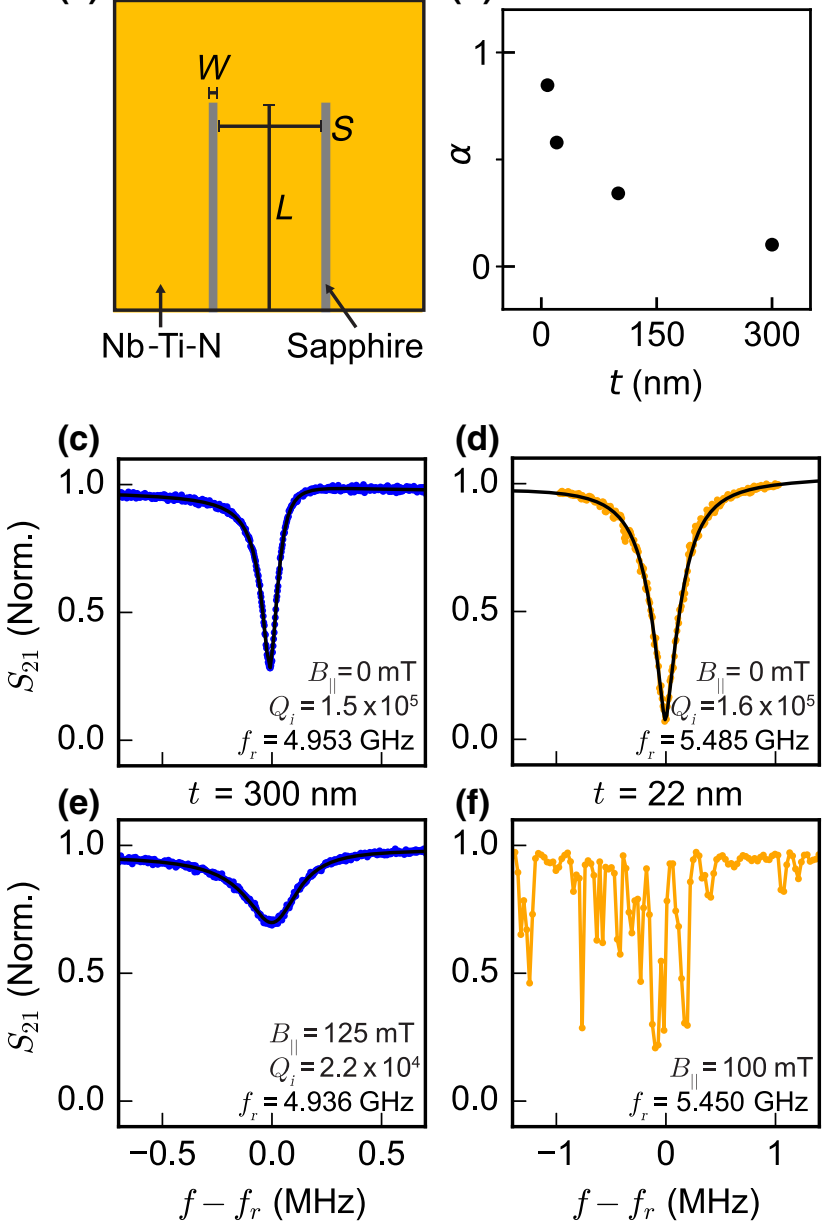

FIG. 2. (a) A top-down schematic showing the shorted end of a $\lambda / 4$ resonator of length $L$ with a central conductor of width $S$ and gap $W$ and no holes. (b) The extracted value of $\alpha$ for each $t$, showing a sharp increase in $\alpha$ for $t \ll \lambda$. (c),(d) $S_{21}$ versus $f$ for resonators with $t=300$ and $22 \mathrm{~nm}$, showing high- $Q_{i}$ stable resonances at $B=0 \mathrm{mT}$. (e),(f) $S_{21}$ versus $f$ for $t=300$ and 22 nm at $B_{\|} \geq 100 \mathrm{mT}$.

At $B=0 \mathrm{~T}$, the transmission spectrum of each sample shows a series of resonances, each corresponding to a frequency-multiplexed resonator. All films display stable resonances, with $Q_{i}$ varying between $10^{4}$ and $10^{5}$. Example resonances for $t=300$ and $22 \mathrm{~nm}$ films are shown in Figs. 2(c) and 2(d), respectively.

A magnetic field $B_{\|}$is then applied parallel to the film using the vector magnet, using the response of the resonator itself to align the magnetic field [49]. This results in the different films displaying markedly different behavior. Measurements at $B_{\|}>100 \mathrm{mT}$ on devices with film thicknesses of $t=100$ and $300 \mathrm{~nm}$ [for $t=300 \mathrm{~nm}$, see Fig. 2(e)] show a homogeneous broadening of their resonances, a reduction in their resonance frequency, and a reduction in $Q_{i}$. This is consistent with increased intrinsic losses and increased inductance due to a high density of 
Abrikosov vortices. At fields above $125 \mathrm{mT}$, the reduction in $Q_{i}$ and the broadening are so extreme that we are unable to resolve the resonances.

In contrast, resonators with film thicknesses of $t=8$ and $22 \mathrm{~nm}$ appear to remain operational up to $B_{\|}=0.25 \mathrm{~T}$ [49]. This represents a significant improvement over the thicker films - although, notably, they instead show inhomogeneous broadening [for $t=22 \mathrm{~nm}$, see Fig. 2(f)], with sharp reductions in $S_{21}$ at multiple points. As the minimum transmission through a resonance $S_{21}^{\mathrm{Min}}=Q_{\mathrm{c}} /\left(Q_{\mathrm{c}}+Q_{i}\right)$ is set by the relative proportions of $Q_{c}$ and $Q_{i}$ [33], the measured response is consistent with a high- $Q$ resonator that is fluctuating in frequency on the same time scale as the measurement. We attribute this behavior to a small number of vortices being pinned in the superconductor, with vortex depinning events occurring on the same time scale as the measurement. While the reduced vortex number is consistent with an enhancement of the critical field, we note that it is still far below the expected theoretical field enhancement of $B_{c_{1}}$ for these films. We attribute this to small misalignments of the magnetic field that interact with the sample geometry and the Meissner effect, locally creating small perpendicular components to the magnetic field, inducing vortices. This interpretation leads to the exciting possibility that if these few vortices created by local inhomogeneities in the magnetic field can be controlled and their detrimental effects mitigated, the enhancement of $B_{c_{1}}$ could be protected.

\section{HOLES}

\section{A. Perpendicular-field dependence}

Defects in the film locally reduce $\Delta_{\mathrm{SC}}$ in the superconductor, creating a potential well in which the energy cost required to break the superconductivity in that region is reduced, providing an additional mechanism for controlling Abrikosov vortices. As it is energetically favorable for the vortex to sit in the defect, it experiences a corresponding restoring force pinning it into the defect, minimizing losses $[33,42,52,53]$. The defects used in this experiment are holes in the superconducting film that are defined by EBL and RIE, meaning that they can be fabricated in the same step as the resonators. The holes are designed to have a smaller diameter than the vortices $(\lambda>d>\xi)$, allowing high hole densities $\rho_{h}$ to be achieved.

We fabricate a sample from a $t=22 \mathrm{~nm}$ film with resonators of different $\rho_{h}$ varying from 0 to $28.8 \mu \mathrm{m}^{-2}$ to determine the effect of holes on resonator performance. The resonators retain a constant CPW geometry, with holes of diameter $d=100 \mathrm{~nm}$ patterned into hexagonal arrays on the central conductor and the edges of the adjacent ground planes. It would take a prohibitive amount of time to use EBL to similarly pattern the entire ground plane, so the remainder of the ground plane is patterned with a rectangular array of larger holes to trap small residual local fluxes [49].

We leave a gap of approximately $1 \mu \mathrm{m}$ to the film edge to ensure that the vortices become pinned far from the current that flows at the edges of the CPW [54], reducing losses from Lorentz effects [33].

The hole densities can be converted to a threshold "critical field" $B_{\text {th }}$ (ranging between 0 and $59.69 \mathrm{mT}$ ) that, when applied perpendicular to the plane of the film, fills each hole with a single Abrikosov vortex [49]. Above $B_{\mathrm{th}}$, additional vortices are no longer strongly pinned by the holes but, instead, only weakly pinned by film defects and interstitial pinning effects.

Field-cooled measurements are performed by applying a perpendicular magnetic field $B_{\perp}$ to the sample above $T_{c} \sim 14 \mathrm{~K}$ of the Nb-Ti-N and then cooling the sample to base temperature before performing transmission measurements to characterize the resonators. At $B=0 \mathrm{~T}$, all resonators are measured with $Q_{i}>10^{5}$ [Fig. 3(a)]. Resonators with a nonzero $\rho_{h}$ trap the few vortices that form due to the local magnetic environment near the CPW, enhancing $Q_{i}$. At higher $\rho_{h}, Q_{i}$ decreases, possibly due to increased losses occurring in the larger metal-vacuum interface area [55].

The behavior of $Q_{i}$ versus $B_{\perp}$ field is plotted in Fig. 3(b) together with the calculated $B_{\text {th }}$ for each hole density. For

(a)
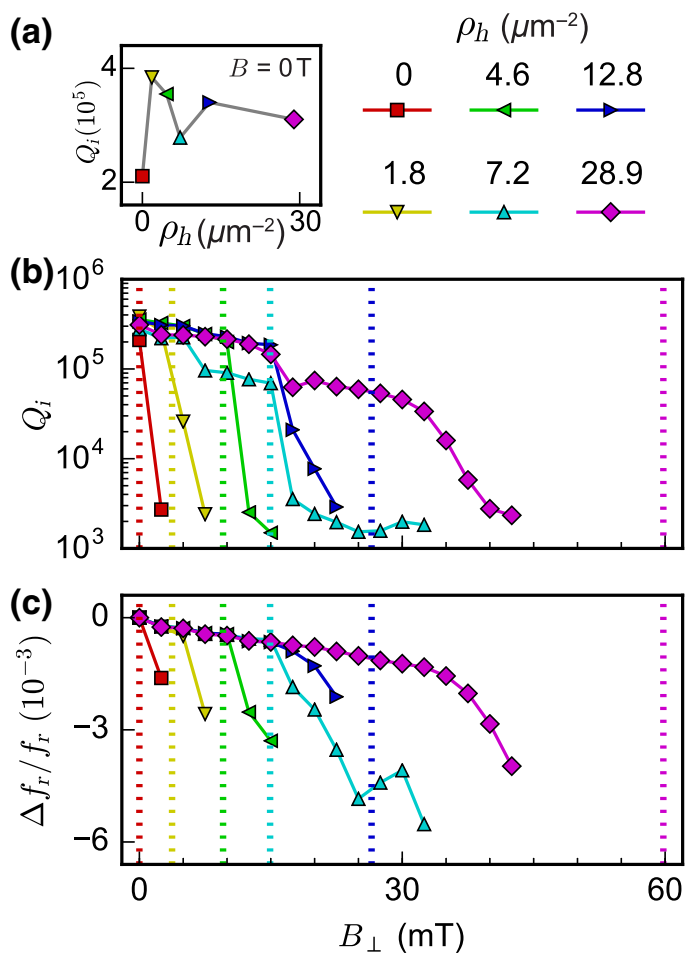

FIG. 3. (a) $Q_{i}$ versus $\rho_{h}$ for field-cooled resonators at $B_{\perp}=$ 0 T. (b) $Q_{i}$ versus $B_{\perp}$ for varying $\rho_{h}$. $B_{\text {th }}$ for each resonator is plotted with a color-matched vertical line. (c) $\Delta f_{r} / f_{r}$ versus $B_{\perp}$ for varying $\rho_{h}$ with $B_{T h}$ plotted as in (b). 
$B<B_{\text {th }}$, each resonator retains a high $Q_{i}$, as all vortices can be trapped in holes. Once every hole is full $\left(B>B_{\mathrm{th}}\right)$, $Q_{i}$ decreases sharply, as additional vortices must create a normal metal core in the SC film, significantly increasing resistive losses. A similar response is observed in the fractional frequency $\Delta f_{r} / f_{r}=\left(f_{r}-f_{r}^{B=0}\right) / f_{r}$ of the resonators [Fig. 3(c)]. For $B<B_{\text {th }}$, the magnetic field increases the rate of Cooper-pair breaking, increasing $L_{k}$ and resulting in a gradual reduction of $f_{r}$ [47]. Once $B>B_{\text {th }}$, the vortices are no longer pinned by the hole lattice and become itinerant, significantly increasing the inductive load on the resonator and resulting in a comparatively large frequency shift.

At $\rho_{h} \geq 12.8 \mu \mathrm{m}^{-2}$, the results start to deviate from the expected response, with both $Q_{i}$ and $\Delta f_{r} / f_{r}$ reducing at a lower $B_{\perp}$ than expected. We attribute this to the vortex density approaching a regime where the supercurrents in the vortices start to overlap and interfere with each other, increasing resistive losses. Unusually, the resonator with highest $\rho_{h}$ experiences a reduction in $Q_{i}$ at approximately $15 \mathrm{mT}$, which is not accompanied by a decrease in $\Delta f_{r} / f_{r}$, as would be expected.

This is suggestive of vortices that do not sit in a pinning site but are also not itinerant. This may occur due to interstitial pinning effects that have been extensively studied previously and could be used to further increase the perpendicular field compatibility of these resonators [56-58].

\section{B. Parallel-field dependence}

To demonstrate that thin films and a low density of holes can be used to increase the resilience of resonators to strong parallel magnetic fields, a sample with 12 resonators of varying CPW dimensions but constant hole density are fabricated from $t=22 \mathrm{~nm}$ films. The holes are $300 \mathrm{~nm}$ in diameter and are arranged in hexagonal lattice around the $\mathrm{CPW}$, with an interhole spacing of $1 \mu \mathrm{m}\left(\rho_{h}=1.2 \mu \mathrm{m}^{-2}\right)$, an intersite spacing of relevance for optical-lithography techniques [Fig. 4(c) inset]. The device is then cooled at $B=0 \mathrm{~T}$ and $S_{21}$ measurements are performed to determine the $Q_{i}$ of all 12 resonators as a function of $B_{\|}$, with $B_{\|}$ applied as in Sec. III, using the response of the resonator for field alignment.

$S_{21}$ versus $f-f_{r}$ for an example resonance at $B_{\|}=$ $0 \mathrm{~T}, 0.1 \mathrm{~T}$, and $5.5 \mathrm{~T}$ [Fig. 4(a)] shows a stable high$Q_{i}$ peak at all fields, a key requirement for their effective use in superconducting circuits. Fitting the resonator response to extract $Q_{i}$ versus $B_{\|}$[Fig. 4(b)] reveals that $Q_{i} \sim 10^{5}$ can be retained up to $B_{\|}=5.5 \mathrm{~T}$. A dip in $Q_{i}$, attributed to coupling to an electron spin resonance (ESR) that increases losses in the cavity, is observed at $B_{\mathrm{ESR}} \sim$ $200 \mathrm{mT}$. This feature is observed in all resonators, with the frequency dependence corresponding to paramagnetic
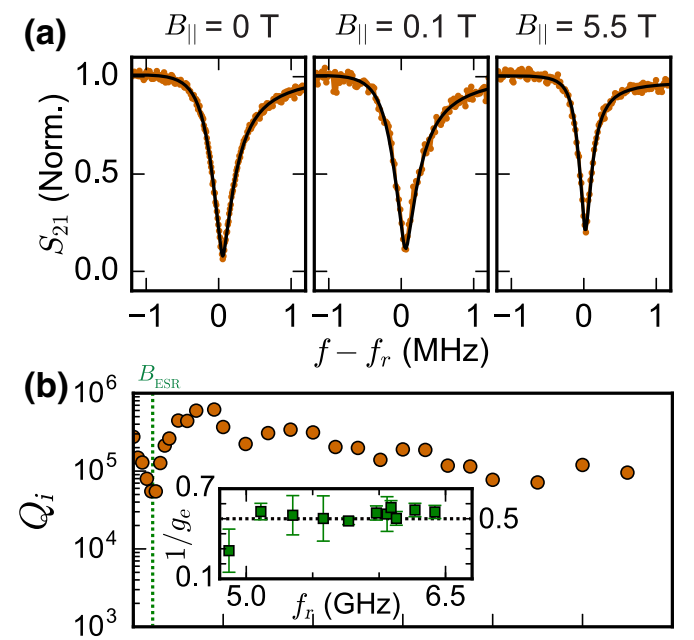

(c)

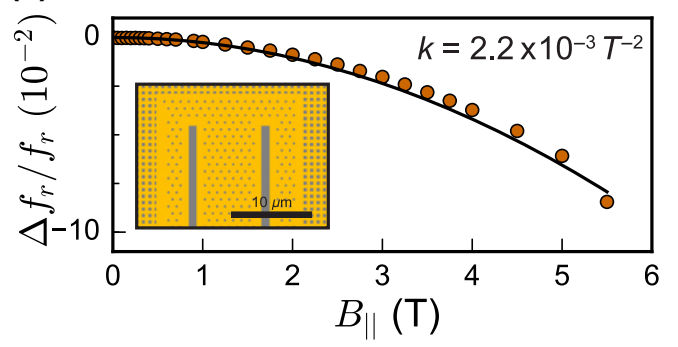

FIG. 4. (a) $S_{21}$ versus $f-f_{r}$ for a typical $t=22 \mathrm{~nm}$ resonator with holes at $B_{\|}=0 \mathrm{~T}, 0.1 \mathrm{~T}$ and $5.5 \mathrm{~T}$. (b) The extracted $Q_{i}$ versus $B_{\|}$. The inset shows the inverse of the extracted $g_{e}$ factor from $Q_{i}$ minima of each resonator as a function of $B_{\|}$. (c) The extracted $\Delta f_{r} / f_{r}$ versus $B_{\|}$. A fit of the data with $\Delta f_{r} / f_{r}=-k B_{\|}^{2}$ gives $k=2.2 \times 10^{-3} \mathrm{~T}^{-2}$. The inset is a diagram showing the distribution of holes around the CPW for one of the resonators.

impurities with a Landé $g$ factor of approximately 2 [Fig. 4(b) inset], as observed in [47].

As $B_{\|}$is increased, the resonator $f_{r}$ decreases [Fig. 4(c)] due to the increased rate of Cooper-pair breaking from the applied $B_{\|}$. It can be modeled as a parabolic decrease in $B_{\|}$ as $\Delta f_{r} / f_{r}=-k B_{\|}^{2}$, with $k=(\pi / 48)\left[t^{2} e^{2} D / \hbar k_{B} T_{c}\right]$ dependent on $t, T_{c}$, and $D$, the electron diffusion constant in Nb-Ti-N. With all other parameters known, we determine $D=0.79 \mathrm{~nm}^{2} \mathrm{~s}^{-1}$ consistent with previous results [47].

This magnetic field dependence is generic to all 12 resonators measured, with a high- $Q_{i}$ resonance measurable up to $B_{\|} \simeq 6 \mathrm{~T}$ [49]. As dielectric losses are known to increase at higher $W / S$ ratios, it may be possible to increase $Q_{i}$ even further by optimizing the CPW geometry. Surprisingly, in all 12 resonators, the maximum $Q_{i}$ in $B_{\|}$does not occur at zero magnetic field, instead occurring at somewhere between $B_{\|}=0.5$ and $1 \mathrm{~T}$. The origin of this $Q_{i}$ enhancement is not clear but we speculate that it may occur due Abrikosov vortices penetrating the superconducting film and acting as quasiparticle traps, reducing the intrinsic losses associated with quasiparticles [59]. At higher magnetic fields, the rate of quasiparticle generation would 
become so great that they would no longer function as effective traps, reducing $Q_{i}$ at high $B_{\|}$as observed.

\section{CHARGE READOUT OF A HYBRID InSb NANOWIRE DEVICE AT $1 \mathrm{~T}$}

DQDs in combination with high-quality-factor SC CPW resonators form a superconductor-semiconductor "hybrid" system allowing for the high-bandwidth sensing of DQD charge configurations in a variety of material systems using techniques from circuit quantum electrodynamics (cQED) $[14,60-62]$. More exotic hybrid systems such as spin qubits [13,25], Majorana box qubits [28], and spintransmon hybrids also require the application of strong parallel magnetic fields, a condition that to date has proven challenging for traditional SC CPW resonators. To demonstrate the importance of our field-resilient patterned SC $\mathrm{CPW}$ for hybrid systems, we integrate a pair of In-Sb nanowires into $a \lambda / 2$ resonator and perform fast charge readout of DQDs at a magnetic field of $B_{\|}=1 \mathrm{~T}$.

The $\lambda / 2$ resonator, the holes, and the electrostatic gates required for forming the DQDs are formed in a single lithography step followed by $\mathrm{SF}_{6} / \mathrm{O}_{2}$ reactive ion etch of a $20-\mathrm{nm}$ Nb-Ti-N film [see Fig. 5(a)]. The holes $(d=$ $150 \mathrm{~nm}$ ) are arranged in a hexagonal lattice with a density of $\rho_{h}=3.2 \mu \mathrm{m}^{-2}$. At each end of the resonator, at the electric field maxima, we deposit a single nanowire on top of a set of five fine gates [Figs. 5(a)-5(c)], using a deterministic deposition technique [63]. A 30-nm layer of sputtered $\mathrm{SiN}_{x}$ electrically isolates the nanowire from the underlying gates. Following sulfur etching to remove the surface oxide from the In-Sb nanowire [64], 150-nm Ti/Al contacts are evaporated on each end of the nanowire to define the electrical potential in the nanowire. One contact is connected directly to the central conductor of the resonator and the other end directly to the ground plane to enhance the coupling between the DQD and the cavity's electric field [13]. By applying voltages to the electrostatic gates, we can control the electron occupation in the nanowire, either turning the device off or tuning it into a single-quantum-dot or DQD configuration.

Transmission measurements of the resonator with each nanowire depleted show a single resonance at frequency $f_{r}=4.922 \mathrm{GHz}$ with loaded quality factor $Q_{l} \simeq 1.8 \times 10^{4}$ [Fig. 5(d)]. The resonator is in the overcoupled regime, with the coupling capacitances of the input and output ports controlling the photon lifetime in the cavity.

The magnetic field $B_{\|}$is applied parallel to the plane of the resonator [Fig. 5(e)], using the response of the resonator itself to align the field (as described in Sec. III). Similarly to the $\lambda / 4$ resonators, $f_{r}$ decreases parabolically in $B_{\|}$due to the increased $L_{k}$ and $Q_{l}$ experiences a decrease at $B_{\mathrm{ESR}} \simeq 200 \mathrm{mT}$, corresponding to paramagnetic impurities coupling to the cavity via electron spin resonance [Fig. 5(e) and inset]. (a)

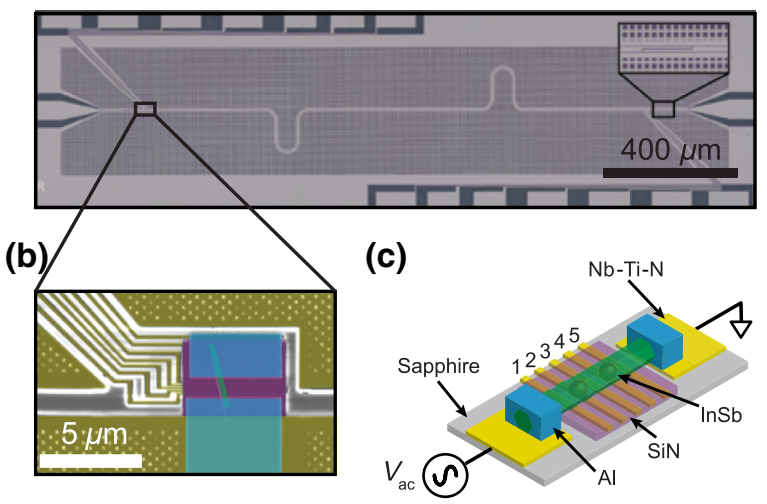

(d)

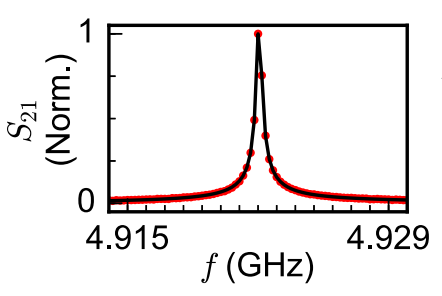

(e)

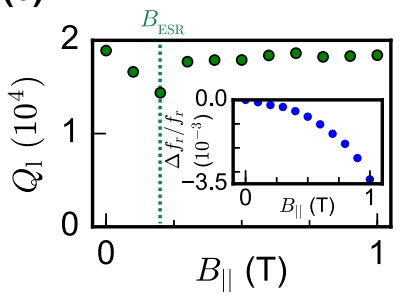

FIG. 5. An optical-microscope image (a), a SEM image (b), and a three-dimensional (3D) diagram (c) of the hybrid device. At each end of the cavity, a single In-Sb nanowire is deposited on local fine gates. $\mathrm{Ti} / \mathrm{Al}$ is used to contact the nanowire to the resonator's central conductor and ground. Local fine gates (1-5) are used to electrostatically define the DQD. Coupling capacitors control the photon lifetime in the cavity. (d) The plot of $S_{21}$ versus $f$ shows a single resonance at $f=4.922 \mathrm{GHz}$ with $Q_{l}=1.8 \times$ $10^{4}$. (e) $Q_{l}$ as a function of $B_{\|}$demonstrates that the resonator is unaffected by strong magnetic fields. The inset shows $\Delta f_{r} / f_{r}$ versus $B_{\|}$, displaying a small parabolic frequency shift.

Once the external magnetic field is aligned and has reached $B_{\|}=1 \mathrm{~T}$, the resonator can be used for tuning the charge occupancy in each of the nanowire DQDs without dc transport. The DQDs are formed by monitoring the microwave response of the resonator and using an applied voltage on outer gates 1 and 5 [Fig. 5(c)] to define a single QD, then using the middle gate 3 to form a DQD. Electron transitions between the dot and lead are a dissipative process that cause a reduction in $Q_{l}$, which can be observed as a reduction in the $S_{21}$ peak amplitude. Additionally, as the interdot tunnel coupling rate of the electrons $t_{c}$ approaches $f_{r}$, the transition coherently couples to the electric field in the cavity via a Jaynes-Cummings-type interaction. This results in a repulsion of the resonator, shifting the resonator frequency and changing the measured phase $\phi[60]$.

Monitoring of the amplitude and phase fluctuations as the plunger gates are varied allows the dot-lead and interdot transitions to be identified and the charge-stability diagrams for each of the DQDs to be measured at $B_{\|}=1 \mathrm{~T}$ [Fig. 6]. For the right DQD, gates 2, 4, and 5 are used to define the dots, while gates 3 and 5 control the electron 

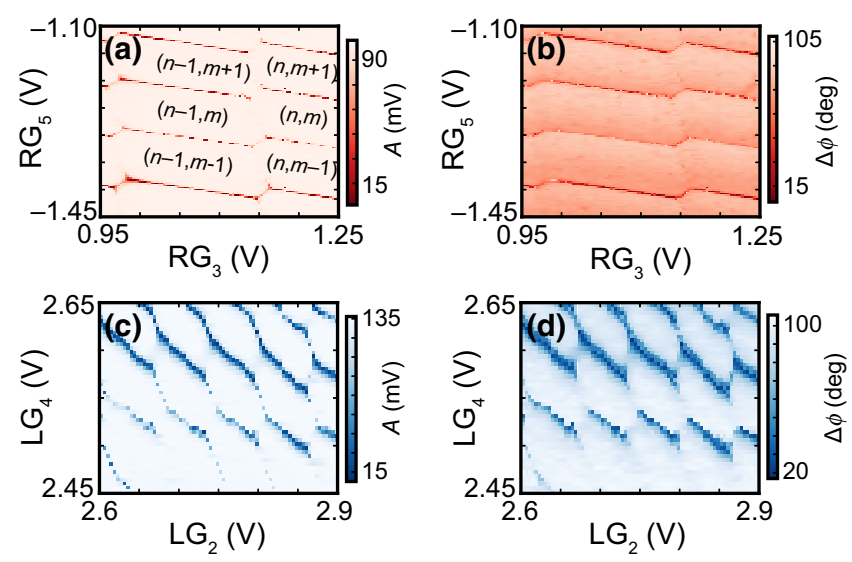

FIG. 6. Measurement of $S_{21}$ at $f_{r}$ at $B=1 \mathrm{~T}$ as $V$ on the fine gates is varied generates charge-stability diagrams of the right (a),(b) and left (c),(d) nanowire DQDs. $\mathrm{RG}_{\mathrm{n}}$ denotes the $\mathrm{n}^{\text {th }}$ gate on the right DQD device. The electron occupation $n(m)$ of the left (right) QD can be modified by tuning the gate voltages.

occupancy [Figs. 6 (a) and 6(b)]. In the left DQD, the electrical confinement is defined by gates 1,3 , and 5 , and the charge occupancy is controlled by gates 2 and 4 [Figs. 6(c) and 6(d)]. For each DQD, the charge occupancy in the left (right) dot is denoted by $n(m)$.

The measurements are performed at $B_{\|}=1 \mathrm{~T}$, an orderof-magnitude improvement over previous studies [13,26], and a magnetic field of relevance for numerous topological QC proposals $[27,28]$ and the investigation of mesoscopic phenomena in hybrid systems under strong magnetic fields [18].

\section{CONCLUSION}

These results indicate that by controlling vortex dynamics in SC CPW resonators, a $Q_{i} \simeq 10^{5}$ can be retained in a perpendicular magnetic field of $B_{\perp} \simeq 20 \mathrm{mT}$. When combined with film-thickness reduction to enhance $B_{c_{1}}$, we establish a reliable fabrication recipe to create SC CPW resonators that can retain a high $Q_{i} \sim 10^{5}$ in strong parallel magnetic fields up to $B_{\|} \simeq 6 \mathrm{~T}$. We demonstrate the importance of these techniques for hybrid systems by coupling a superconducting resonator to DQDs electrostatically defined in In-Sb nanowires. Using high-frequency measurement techniques, we demonstrate the device operation and determine the charge-stability diagrams of two DQDs at $B_{\|}=1 \mathrm{~T}$, a magnetic field of relevance for mesoscopic physics studies and semiconducting, topological, and hybrid QC schemes.

\section{ACKNOWLEDGMENTS}

We thank N. Samkharadze, C. Dickel, F. Lüthi, N. Langford, and L. DiCarlo for useful discussions. This work has been supported by the Netherlands Organisation for
Scientific Research (NWO/OCW), as part of the Frontiers of Nanoscience (NanoFront) program, the European Research Council (ERC), and Microsoft Quantum Lab Delft.

[1] M. A. Castellanos-Beltran and K. W. Lehnert, Widely tunable parametric amplifier based on a superconducting quantum interference device array resonator, Appl. Phys. Lett. 91, 083509 (2007).

[2] E. A. Tholén, A. Ergül, E. M. Doherty, F. M. Weber, F. Grégis, and D. B. Haviland, Nonlinearities and parametric amplification in superconducting coplanar waveguide resonators, Appl. Phys. Lett. 90, 253509 (2007).

[3] B. A. Mazin, P. K. Day, H. G. LeDuc, A. Vayonakis, and J. Zmuidzinas, Superconducting Kinetic Inductance Photon Detectors (The Society Of Photo-optical Instrumentation Engineers, Waikoloa, HI, USA, 2002), p. 283.

[4] P. K. Day, H. G. LeDuc, B. A. Mazin, A. Vayonakis, and J. Zmuidzinas, A broadband superconducting detector suitable for use in large arrays, Nature 425, 817 (2003).

[5] G. Vardulakis, S. Withington, D. J. Goldie, and D. M. Glowacka, Superconducting kinetic inductance detectors for astrophysics, Meas. Sci. Technol. 19, 015509 (2008).

[6] H. Hattermann, D. Bothner, L. Y. Ley, B. Ferdinand, D. Wiedmaier, L. Sárkány, R. Kleiner, D. Koelle, and J. Fortágh, Coupling ultracold atoms to a superconducting coplanar waveguide resonator, Nat. Commun. 8, 2254 (2017).

[7] Y. Kubo, F. R. Ong, P. Bertet, D. Vion, V. Jacques, D. Zheng, A. Dréau, J. F. Roch, A. Auffeves, F. Jelezko, J. Wrachtrup, M. F. Barthe, P. Bergonzo, and D. Esteve, Strong Coupling of a Spin Ensemble to a Superconducting Resonator, Phys. Rev. Lett. 105, 140502 (2010).

[8] R. Amsüss, C. Koller, T. Nöbauer, S. Putz, S. Rotter, K. Sandner, S. Schneider, M. Schramböck, G. Steinhauser, H. Ritsch, J. Schmiedmayer, and J. Majer, Cavity QED with Magnetically Coupled Collective Spin States, Phys. Rev. Lett. 107, 060502 (2011).

[9] V. Ranjan, G. De Lange, R. Schutjens, T. Debelhoir, J. P. Groen, D. Szombati, D. J. Thoen, T. M. Klapwijk, R. Hanson, and L. Dicarlo, Probing Dynamics of an Electron-Spin Ensemble via a Superconducting Resonator, Phys. Rev. Lett. 110, 067004 (2013).

[10] C. A. Regal, J. D. Teufel, and K. W. Lehnert, Measuring nanomechanical motion with a microwave cavity interferometer, Nat. Phys. 4, 555 (2008).

[11] J. D. Teufel, C. A. Regal, and K. W. Lehnert, Prospects for cooling nanomechanical motion by coupling to a superconducting microwave resonator, New J. Phys. 10, 095002 (2008).

[12] G. Burkard and A. Imamoglu, Ultra-long-distance interaction between spin qubits, Phys. Rev. B 74, 041307 (2006).

[13] K. D. Petersson, L. W. McFaul, M. D. Schroer, M. Jung, J. M. Taylor, A. A. Houck, and J. R. Petta, Circuit quantum electrodynamics with a spin qubit, Nature 490, 380 (2012). 
[14] J. J. Viennot, M. C. Dartiailh, A. Cottet, and T. Kontos, Coherent coupling of a single spin to microwave cavity photons, Science 349, 408 (2015).

[15] Y.-Y. Liu, J. Stehlik, C. Eichler, M. J. Gullans, J. M. Taylor, and J. R. Petta, Semiconductor double quantum dot micromaser, Science 347, 285 (2015).

[16] G. De Lange, B. Van Heck, A. Bruno, D. J. Van Woerkom, A. Geresdi, S. R. Plissard, E. P. A. M. Bakkers, A. R. Akhmerov, and L. DiCarlo, Realization of Microwave Quantum Circuits Using Hybrid Superconducting-Semiconducting Nanowire Josephson Elements, Phys. Rev. Lett. 115, 127002 (2015).

[17] T. W. Larsen, K. D. Petersson, F. Kuemmeth, T. S. Jespersen, P. Krogstrup, J. Nygrd, and C. M. Marcus, Semiconductor-Nanowire-Based Superconducting Qubit, Phys. Rev. Lett. 115, 127001 (2015).

[18] J. G. Kroll, W. Uilhoorn, K. L. van der Enden, D. de Jong, K. Watanabe, T. Taniguchi, S. Goswami, M. C. Cassidy, and L. P. Kouwenhoven, Magnetic field compatible circuit quantum electrodynamics with graphene Josephson junctions, Nat. Commun. 9, 4615 (2018).

[19] A. Blais, R. S. Huang, A. Wallraff, S. M. Girvin, and R. J. Schoelkopf, Cavity quantum electrodynamics for superconducting electrical circuits: An architecture for quantum computation, Phys. Rev. A 69, 062320 (2004).

[20] A. Wallraff, D. I. Schuster, A. Blais, L. Frunzio, R. S. Huang, J. Majer, S. Kumar, S. M. Girvin, and R. J. Schoelkopf, Strong coupling of a single photon to a superconducting qubit using circuit quantum electrodynamics, Nature 431, 162 (2004).

[21] A. Wallraff, D. I. Schuster, A. Blais, L. Frunzio, J. Majer, M. H. Devoret, S. M. Girvin, and R. J. Schoelkopf, Approaching Unit Visibility for Control of a Superconducting Qubit with Dispersive Readout, Phys. Rev. Lett. 95, 060501 (2005).

[22] J. Majer, J. M. Chow, J. M. Gambetta, J. Koch, B. R. Johnson, J. A. Schreier, L. Frunzio, D. I. Schuster, A. A. Houck, A. Wallraff, A. Blais, M. H. Devoret, S. M. Girvin, and R. J. Schoelkopf, Coupling superconducting qubits via a cavity bus, Nature 449, 443 (2007).

[23] A. Stockklauser, P. Scarlino, J. V. Koski, S. Gasparinetti, C. K. Andersen, C. Reichl, W. Wegscheider, T. Ihn, K. Ensslin, and A. Wallraff, Strong Coupling Cavity QED with Gate-Defined Double Quantum Dots Enabled by a High Impedance Resonator, Phys. Rev. X 7, 011030 (2017).

[24] A. J. Landig, J. V. Koski, P. Scarlino, U. C. Mendes, A. Blais, C. Reichl, W. Wegscheider, A. Wallraff, K. Ensslin, and T. Ihn, Coherent spin-qubit photon coupling, Nature 560, 179 (2017).

[25] X. Mi, M. Benito, S. Putz, D. M. Zajac, J. M. Taylor, G. Burkard, and J. R. Petta, A coherent spin-photon interface in silicon, Nature 555, 599 (2018).

[26] N. Samkharadze, G. Zheng, N. Kalhor, D. Brousse, A. Sammak, U. C. Mendes, A. Blais, G. Scappucci, and L. M. K. Vandersypen, Strong spin-photon coupling in silicon, Science 359, 1123 (2018).

[27] T. Hyart, B. Van Heck, I. C. Fulga, M. Burrello, A. R. Akhmerov, and C. W. J. Beenakker, Flux-controlled quantum computation with Majorana fermions, Phys. Rev. B 88, 035121 (2013).
[28] S. Plugge, A. Rasmussen, R. Egger, and K. Flensberg, Majorana box qubits, New J. Phys. 19, 012001 (2017).

[29] J. Koch, T. Yu, J. Gambetta, A. A. Houck, D. I. Schuster, J. Majer, A. Blais, M. H. Devoret, S. M. Girvin, and R. J. Schoelkopf, Charge-insensitive qubit design derived from the Cooper pair box, Phys. Rev. A 76, 042319 (2007).

[30] S. P. Harvey, C. G. L. Bøttcher, L. A. Orona, S. D. Bartlett, A. C. Doherty, and A. Yacoby, Coupling two spin qubits with a high-impedance resonator, Phys. Rev. B 97, 235409 (2018).

[31] J. Gao, M. Daal, A. Vayonakis, S. Kumar, J. Zmuidzinas, B. Sadoulet, B. A. Mazin, P. K. Day, and H. G. Leduc, Experimental evidence for a surface distribution of twolevel systems in superconducting lithographed microwave resonators, Appl. Phys. Lett. 92, 152505 (2008).

[32] R. Barends, J. Wenner, M. Lenander, Y. Chen, R. C. Bialczak, J. Kelly, E. Lucero, P. O’Malley, M. Mariantoni, D. Sank, H. Wang, T. C. White, Y. Yin, J. Zhao, A. N. Cleland, J. M. Martinis, and J. J. A. Baselmans, Minimizing quasiparticle generation from stray infrared light in superconducting quantum circuits, Appl. Phys. Lett. 99, 113507 (2011).

[33] C. Song, PhD thesis, School Syracuse University, 2011.

[34] A. Megrant, C. Neill, R. Barends, B. Chiaro, Y. Chen, L. Feigl, J. Kelly, E. Lucero, M. Mariantoni, P. J. J. O’Malley, D. Sank, A. Vainsencher, J. Wenner, T. C. White, Y. Yin, J. Zhao, C. J. Palmstrøm, J. M. Martinis, and A. N. Cleland, Planar superconducting resonators with internal quality factors above one million, Appl. Phys. Lett. 100, 113510 (2012).

[35] A. Bruno, G. De Lange, S. Asaad, K. L. Van Der Enden, N. K. Langford, and L. Dicarlo, Reducing intrinsic loss in superconducting resonators by surface treatment and deep etching of silicon substrates, Appl. Phys. Lett. 106, 182601 (2015).

[36] V. E. Calado, S. Goswami, G. Nanda, M. Diez, A. R. Akhmerov, K. Watanabe, T. Taniguchi, T. M. Klapwijk, and L. M. K. Vandersypen, Ballistic Josephson junctions in edge-contacted graphene, Nat. Nanotechnol. 10, 761 (2015).

[37] D. J. Van Woerkom, A. Geresdi, and L. P. Kouwenhoven, One minute parity lifetime of a NbTiN Cooper-pair transistor: Supplementary information, Nat. Phys. 11, 547 (2015).

[38] M. R. Vissers, J. Gao, D. S. Wisbey, D. A. Hite, C. C. Tsuei, A. D. Corcoles, M. Steffen, and D. P. Pappas, Low loss superconducting titanium nitride coplanar waveguide resonators, Appl. Phys. Lett. 97, 232509 (2010).

[39] V. Singh, B. H. Schneider, S. J. Bosman, E. P. J. Merkx, and G. A. Steele, Molybdenum-rhenium alloy based high$Q$ superconducting microwave resonators, Appl. Phys. Lett. 105, 222601 (2014).

[40] S. Kwon, A. Fadavi Roudsari, O. W. B. Benningshof, Y.-C. Tang, H. R. Mohebbi, I. A. J. Taminiau, D. Langenberg, S. Lee, G. Nichols, D. G. Cory, and G.-X. Miao, Magnetic field dependent microwave losses in superconducting niobium microstrip resonators, J. Appl. Phys. 124, 033903 (2018).

[41] A. Ghirri, C. Bonizzoni, D. Gerace, S. Sanna, A. Cassinese, and $\mathrm{M}$. Affronte, $\mathrm{YBa}_{2} \mathrm{Cu}_{3} \mathrm{O}_{7}$ microwave resonators 
for strong collective coupling with spin ensembles, Appl. Phys. Lett. 106, 184101 (2015).

[42] D. Bothner, T. Gaber, M. Kemmler, D. Koelle, and R. Kleiner, Improving the performance of superconducting microwave resonators in magnetic fields, Appl. Phys. Lett. 98, 102504 (2011).

[43] G. Stan, S. B. Field, and J. M. Martinis, Critical Field for Complete Vortex Expulsion from Narrow Superconducting Strips, Phys. Rev. Lett. 92, 097003 (2004).

[44] K. H. Kuit, J. R. Kirtley, W. van der Veur, C. G. Molenaar, F. J. G. Roesthuis, A. G. P. Troeman, J. R. Clem, H. Hilgenkamp, H. Rogalla, and J. Flokstra, Vortex trapping and expulsion in thin-film $\mathrm{YBa}_{2} \mathrm{Cu}_{3} \mathrm{O}_{7}$ strips, Phys. Rev. B 77, 134504 (2008).

[45] S. E. de Graaf, A. V. Danilov, A. Adamyan, and S. E. Kubatkin, A near-field scanning microwave microscope based on a superconducting resonator for low power measurements, Rev. Sci. Instrum. 84, 023706 (2013).

[46] S. E. de Graaf, A. V. Danilov, A. Adamyan, T. Bauch, and S. E. Kubatkin, Magnetic field resilient superconducting fractal resonators for coupling to free spins, J. Appl. Phys. 112, 123905 (2012).

[47] N. Samkharadze, A. Bruno, P. Scarlino, G. Zheng, D. P. Divincenzo, L. Dicarlo, and L. M. K. Vandersypen, HighKinetic-Inductance Superconducting Nanowire Resonators for Circuit QED in a Magnetic Field, Phys. Rev. Appl. 5, 044004 (2016).

[48] M. S. Khalil, M. J. A. Stoutimore, F. C. Wellstood, and K. D. Osborn, An analysis method for asymmetric resonator transmission applied to superconducting devices, J. Appl. Phys. 111, 054510 (2012).

[49] See the Supplemental Material at http://link.aps.org/supple mental/10.1103/PhysRevApplied.11.064053 for the device parameters, the fitting procedure, the magnetic field alignment, the thickness dependence in a magnetic field, calculation of the threshold field, and the full data set of resonators in a parallel magnetic field.

[50] D. J. Thoen, B. G. C. Bos, E. A. F. Haalebos, T. M. Klapwijk, J. J. A. Baselmans, and A. Endo, Superconducting NbTiN Thin Films With Highly Uniform Properties over a $100 \mathrm{~mm}$ Wafer, IEEE Trans. Appl. Supercond. 27, 1 (2017).

[51] R. Barends, PhD thesis, School Delft University of Technology, 2009.

[52] I. Nsanzineza and B. L. T. Plourde, Trapping a Single Vortex and Reducing Quasiparticles in a Superconducting Resonator, Phys. Rev. Lett. 113, 117002 (2014).

[53] C.-S. Lee, B. Jankó, I. Derényi, and A.-L. Barabási, Reducing vortex density in superconductors using the ratchet effect, Nature 400, 337 (1999).
[54] D. Bothner, T. Gaber, M. Kemmler, D. Koelle, R. Kleiner, S. Wünsch, and M. Siegel, Magnetic hysteresis effects in superconducting coplanar microwave resonators, Phys. Rev. B 86, 014517 (2012).

[55] W. Woods, G. Calusine, A. Melville, A. Sevi, E. Golden, D. K. Kim, D. Rosenberg, J. L. Yoder, and W. D. Oliver, Determining interface dielectric losses in superconducting coplanar waveguide resonators, arXiv:1808.10347 (2018).

[56] M. L. Latimer, G. R. Berdiyorov, Z. L. Xiao, W. K. Kwok, and F. M. Peeters, Vortex interaction enhanced saturation number and caging effect in a superconducting film with a honeycomb array of nanoscale holes, Phys. Rev. B 85, 12505 (2012).

[57] M. Velez, D. Jaque, J. I. Martín, M. I. Montero, I. K. Schuller, and J. L. Vicent, Vortex lattice channeling effects in $\mathrm{Nb}$ films induced by anisotropic arrays of mesoscopic pinning centers, Phys. Rev. B 65, 104511 (2002).

[58] V. V. Moshchalkov, M. Baert, V. V. Metlushko, E. Rosseel, M. J. Van Bael, K. Temst, Y. Bruynseraede, and R. Jonckheere, Pinning by an antidot lattice: The problem of the optimum antidot size, Phys. Rev. B 57, 3615 (1998).

[59] I. Nsanzineza and B. Plourde, Trapping a Single Vortex and Reducing Quasiparticles in a Superconducting Resonator, Phys. Rev. Lett. 113, 117002 (2014).

[60] T. Frey, P. J. Leek, M. Beck, A. Blais, T. Ihn, K. Ensslin, and A. Wallraff, Dipole Coupling of a Double Quantum Dot to a Microwave Resonator, Phys. Rev. Lett. 108, 046807 (2012).

[61] V. Ranjan, G. Puebla-Hellmann, M. Jung, T. Hasler, A. Nunnenkamp, M. Muoth, C. Hierold, A. Wallraff, and C. Schönenberger, Clean carbon nanotubes coupled to superconducting impedance-matching circuits, Nat. Commun. 6, 7165 (2015).

[62] R. Wang, R. S. Deacon, D. Car, E. P. A. M. Bakkers, and K. Ishibashi, InSb nanowire double quantum dots coupled to a superconducting microwave cavity, Appl. Phys. Lett. 108, 203502 (2016).

[63] K. Flöhr, M. Liebmann, K. Sladek, H. Y. Günel, R. Frielinghaus, F. Haas, C. Meyer, H. Hardtdegen, T. Schäpers, D. Grützmacher, and M. Morgenstern, Manipulating InAs nanowires with submicrometer precision, Rev. Sci. Instrum. 82, 113705 (2011).

[64] Ö. Gül, H. Zhang, F. K. De Vries, J. Van Veen, K. Zuo, V. Mourik, S. Conesa-Boj, M. P. Nowak, D. J. Van Woerkom, M. Quintero-Pérez, M. C. Cassidy, A. Geresdi, S. Koelling, D. Car, S. R. Plissard, E. P. A. M. Bakkers, and L. P. Kouwenhoven, Hard superconducting gap in InSb nanowires, Nano Lett. 17, 2690 (2017). 